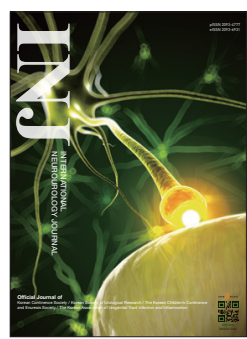

\title{
Letters Published in the June Issue
}

\author{
Hong Sang Moon (ib https://orcid.org/0000-0003-2101-1019 \\ Department of Urology, Hanyang University College of Medicine, Seoul, Korea \\ E-mail: moonuro@hanyang.ac.kr
}

International Neurourology Journal (INJ) will publish a total of 4 letters this month. Two of them are letters about the article entitled "Role of Transcutaneous Electrical Nerve Stimulation in Treating Children With Overactive Bladder From Pooled Analysis of 8 Randomized Controlled Trials" published in the March issue 2020 of INJ, and the others are the letters about the article "The Urine Microbiome of Healthy Men and Women Differs by Urine Collection Method," and the article "coronavirus disease 2019 (COVID-19)" which were also published in the March issue 2020 of INJ [1-3].

In the absence of a gold standard for the diagnosis or treatment of a disease, doctors have difficulties diagnosing and treating patients. In order to solve these problems, clinical studies are conducted. Clinical studies have various possible methodologies, and randomized controlled trials (RCTs) are especially reliable. A meta-analysis is a collection and analysis of the results of RCTs on a given topic [4]. Meta-analyses have the advantage of overcoming the limitations of individual studies and obtaining generalizable and objective results. However, since meta-analyses are studies of existing studies, a potential problem is that they are affected by the quality of existing studies. In addition, meta-analyses have been criticized for mixing heterogeneous studies together and analyzing them, as if comparing apples to oranges. In order to overcome these problems, researchers must produce integrated and objective results by minimizing the errors that can occur in the meta-analysis process. In my opinion, the 2 letters about the article "Role of Transcutaneous Electrical Nerve Stimulation in Treating Children With Overactive Bladder From Pooled Analysis of 8 Randomized Controlled Trials" reflect an effort to overcome the problems that may occur during meta-analysis [5,6].

The results of research published on the human microbiome are often difficult to compare because of differences in study design, and many problems remain to be solved. The letter that Professor Lee sent regarding the article "The Urine Microbiome of Healthy Men and Women Differs by Urine Collection Method" contains an insightful description of future challenges facing research into the human urinary microbiome [7].

Professor Kim sent the letter about health disparities during the COVID-19 pandemic [8]. I hope the day will come when COVID-19 disappears quickly on Earth.

On behalf of the Editorial Board of INJ, I would like to thank all the authors who wrote letters for the June issue of INJ.

- Conflict of Interest: No potential conflict of interest relevant to this article was reported.

\section{REFERENCES}

1. Cui H, Yao Y, Xu Z, Gao Z, Wu J, Zhou Z, et al. Role of transcutaneous electrical nerve stimulation in treating children with overactive bladder from pooled analysis of 8 randomized controlled trials. Int Neurourol J 2020;24:84-94.

2. Pohl HG, Groah SL, Perez-Losada M, Ljungberg I, Sprague BM, Chandal N, et al. The urine microbiome of healthy men and women differs by urine collection method. Int Neurourol J 2020;24:4151.

3. Kim KH, COVID-19. Int Neurourol J 2020;24:1-1.

4. Kim DK, Lee JY, Jung JH, Kim JH, Hah YS, Hong CH, et al. Alpha-1 adrenergic receptor blockers for the treatment of lower uri- 
nary tract symptoms in women: a systematic review and metaanalysis. Int Neurourol J 2019;23:56-68.

5. Beloy IC, Gonz MG, Argibay IS. Commentary on "Role of transcutaneous electrical nerve stimulation in treating children with overactive bladder from pooled analysis of 8 randomized controlled trials". Int Neurourol J 2020;24:185-6.

6. Zhou Z, Cui Y. Reply to commentary on "Role of transcutaneous electrical nerve stimulation in treating children with overactive bladder from pooled analysis of 8 randomized controlled trials". Int Neurourol J 2020;24:187-8.

7. Lee SJ, Commentary on "The urine microbiome of healthy men and women differs by urine collection method". Int Neurourol J 2020;24:182-4.

8. Kim J. Equality, inclusion, and diversity in healthcare during the COVID-19 pandemic. Int Neurourol J 2020;24:180-1 